\title{
Chemokine-receptor activation by env determines the mechanism of death in HIV-infected and uninfected T Iymphocytes
}

\author{
Stacey R. Vlahakis, ${ }^{1}$ Alicia Algeciras-Schimnich, ${ }^{2}$ German Bou, ${ }^{2}$ Carrie J. Heppelmann, ${ }^{3}$ \\ Angelina Villasis-Keever, ${ }^{2}$ Ronald G. Collman, ${ }^{4}$ and Carlos V. Paya ${ }^{1,2,3}$ \\ ${ }^{1}$ Division of Infectious Diseases, \\ ${ }^{2}$ Department of Immunology, and \\ ${ }^{3}$ Division of Experimental Pathology, M ayo Clinic, Rochester, Minnesota, USA \\ ${ }^{4}$ Department of M edicine, University of Pennsylvania School of M edicine, Philadel phia, Pennsylvania, USA
}

Address correspondenceto: Carlos V. Paya, Division of Infectious Diseases, M ayo Clinic, 200 First Street SW/GU-501, Rochester, Minnesota 55905, USA. Phone: (507) 284-3747; Fax: (507) 284-3757; E-mail: paya@mayo.edu.

Received for publication August 18, 2000, and accepted in revised form December 5, 2000.

There is considerable confusion concerning the mechanism of Iymphocyte death during HIV infection. During the course of HIV infection, M -tropic viruses (R5) that use CCR 5 chemokine coreceptors frequently evolve to T-tropic viruses (X4) that use CXCR4 receptors. In this study we show that activation of the CD 4 or CCR 5 receptor by R5 HIVenv causes a caspase 8-dependent death of both uninfected and infected CD 4 T cells. In contrast, CXCR 4 activation by X4 HIVenv induces a caspase-independent death of both uninfected CD 4 and CD $8 T$ cells and infected CD 4 cells. These results suggest that activation of the chemokine receptor by HIVenv determines the mechanism of death for both infected and uninfected T Iymphocytes.

J. Clin. Invest. 107:207-215 (2001).

\section{Introduction}

Enhanced depletion of mature peripheral $T$ cells is in part responsible for the progressive decline of CD 4 and, in the latestages of HIV infection, of CD $8 \mathrm{~T}$ cells. M ultiple mechanisms have been proposed to explain the depletion of peripheral $T$ cells that fall into two broad categories overall: "indirect” death of uninfected T cells by apoptosis secondary to their aberrant activation and "direct" killing of HIV-infected T cells. Furthermore, it has been suggested that thesetwo forms of T-cell death are mediated by different molecular mechanisms. Uninfected CD 4 T cells may undergo apoptosis after their aberrant activation by soluble or membranebound HIVenv by CD 4 (1-5). This env/ CD 4-dependent activation of the $T$ Iymphocyte by HIVenv sensitizes uninfected cells to a Fas/FasL-mediated apoptosis, which is a caspase-dependent process $(6,7)$. HIVenv also mediates apoptosis of active HIV-infected T cells $(4,8)$. In contrast, this direct HIV-mediated T-cell death is independent of caspaseactivation (9). Furthermore, HIV-induced cell death of theinfected cell is not antagonized by antiapoptotic molecules such as bcl-2 (10).

During the early stages of HIV infection, M-tropic or R5 strains predominate. These strains use both the $C C R$ and $C D 4$ receptors for viral entry and are clinically associated with a decline in CD 4, but not CD 8 T cells. In later stages of infection, HIV evolves into the Ttropic or X4 strain, which requires CXCR4 as its coreceptor for viral entry. This stage is clinically characterized by depletion of CD 4 T cells and, importantly, depletion of CD 8 T cells (11-16). The apparent differ- ent behavior of $\mathrm{R} 5$ and $\mathrm{X} 4$ strains with regard to $\mathrm{CD} 4$ $T$-cell depletion has been confirmed recently in SHIVinfected macaques (17). While the underlying molecular basis for this observation is unknown, it suggests that thechemokineHIV coreceptor, and henceHIVenv, may play a major rolein T-cell death.

In this study we have investigated whether HIVenv is responsible for both the death of primary-uninfected and HIV-infected T cells by studying the role of the HIVenv/chemokine coreceptor interaction in mediating T-cell death.

\section{Methods}

Cells, cell-receptor stimulation, and reagents. PBM Cs were isolated by Ficoll-H ypaquefrom healthy blood donors, and CD 4 and CD 8 cells werepurified by negativedepletion using magnetic columns (Stem Cell Technologies Inc., Vancouver, British Columbia, Canada) as described previously (6), yielding CD $4\left(99 \% \mathrm{CD}^{+}\right.$and $\left.98 \% \mathrm{CD} 4\right)$ and CD $8\left(98 \% \mathrm{CD}^{+}\right.$and $\left.91 \% \mathrm{CD} 8\right)$ T-lymphocytepopulations, as determined by using flow cytometry. Whole blood from CCR 532 -mutant donors was a generous gift from Ronald Collman (University of Pennsylvania, Philadelphia, Pennsylvania, USA).

Lymphocyte receptors were cross-linked with Ab's as described previously (6). Primary Ab was used at $5 \mu \mathrm{g} / 2$ $\times 10^{6} \mathrm{cell} / \mathrm{ml}$ for 1 hour at $4^{\circ} \mathrm{C}$. Anti-CD 4 Ab (Leu-3a) and isotype IgG controls were purchased from Becton Dickinson Immunocytometry Systems (San Diego, California, USA). Anti-CXCR4 $12 \mathrm{G} 5$ and anti-CCR5 MAB183 Ab's were obtained form the NIH AIDS 
Research and Reference Program (Bethesda, $M$ aryland, USA). Recombinant soluble(s) X4 (HIV LAI ) gp120 was purchased from Protein Sciences Corp. (Bethesda, M aryland, USA) and incubated with lymphocytes at a concentration of $5 \mu \mathrm{g} / 2 \times 10^{6} \mathrm{cell} / \mathrm{ml}$ for 1 hour. Primary 92U g20.9 X4 env was generously donated by Claudia Cicola (NIH) and used at $20 \mathrm{nM}$. In some experiments, results wereconfirmed using $0.5 \mu \mathrm{g} / 2 \times 10^{6} \mathrm{cells}$. Ab- or gp120-treated primary $T$ cells were then counted and transferred to 96-well plates in triplicate at a concentration of $10^{5} \mathrm{cells} /$ well. Cells assessed for Fas-mediated apoptosis werefurther treated with anti-Fas cross-linking IgM $\mathrm{Ab}(\mathrm{CH}-11)$ (Upstate Biotechnology Inc., Lake Placid, N ew York, USA) for 18 hours at $37^{\circ} \mathrm{C}$. In some experiments, CD 4 and CD $8 T$ cells werepretreated with the pancaspase inhibitor Z-VAD (Calbiochem-Novabiochem Corp., San Diego, California, USA), the caspase 8 inhibitor Z-IETD (Calbiochem-N ovabiochem Corp.), or caspase 9 inhibitor Z-LEHD (CalbiochemNovabiochem Corp.) at a concentration of $20 \mu \mathrm{M}$ at $37^{\circ} \mathrm{C}$ for 1 hour. Pertussis toxin (Calbiochem-N ovabiochem Corp.) was used at a concentration of $1 \mu \mathrm{g} / \mathrm{mL}$ at $37^{\circ} \mathrm{C}$ for 1 hour. SD F1 $\alpha$ (Becton Dickinson Immunocytometry Systems) was used at $250 \mathrm{nM}$ when incubated with primarylymphocytes for 1 hour at $37^{\circ} \mathrm{C}$. MIP1 $\beta$ (Becton Dickinson Immunocytometry Systems) was incubated with lymphocytes for 1 hour at $37^{\circ} \mathrm{C}$ at 1 $\mu \mathrm{g} / \mathrm{ml}$. Soluble sCD 4 (NIH AIDS Research and Reference Program) was used at $2-100 \mu \mathrm{g} / \mathrm{ml}$ for 30 minutes at $4^{\circ} \mathrm{C}$ when incubated with soluble gp120 or 293Tenv-expressing cells. IL-4 (R\&D Systems Inc., Minneapolis, M innesota, USA) was used at $10 \mathrm{ng}$ for 48 hours at $37^{\circ} \mathrm{C}$. Azidothymidine (AZT) was purchased from Sigma Chemical Co. (St. Louis, M issouri, USA).

Cell-mixing experiments, cell-death analysis, and flow cytometry. 293T cells were transiently transfected by Fugene (Roche M olecular Biochemicals, Indianapolis, Indiana, USA) according to manufacturer's specification, with an HIV-rev-expressing plasmid (pREV) and an empty vector (SFFV), or SFFV plasmid expressing the envelope CDNA of the R5 strain $\mathrm{HIV}_{\mathrm{JRFL}}$, or the X4 strain HIV $\mathrm{VXX}_{\text {HX2 }}$ (provided by D. Littman, Skirball Institute, N ew York University M edical Center, N ew York, N ew York, USA). After 48 hours, the 293T cells were harvested and mixed in suspension with CD 4 or CD 8 T cells at $10^{6} 293 \mathrm{~T}$ cells $/ 2 \times 10^{6}$ /ymphocytes in duplicatein a 24-well platefor 2 hours. T cells werethen harvested, counted, and plated in triplicate in 96-well plates at $10^{5} \mathrm{~T}$ cells/ well for 18 hours. To determine expression of env, transfected 293T cells werelysed, separated by SDS-PAGE, transferred to PVDF membranes, and immunoblotted with anti-gp120 Ab's (NIH AIDS Research and Reference Program).

The percentage of cell mortality was calculated using Trypan bluedye exclusion as follows: 1 - (total number of cells viable on day $2 /$ total number of cells viable on day 1 immediately after stimulation) $\times 100$. Cell death was al so confirmed in each experiment using two flowcytometry-based methods: cells with reduced FSC and increased hypodiploid DN A content following propidium iodidestaining (18). For theflow-cytometric methods, thepercentage of cell death is presented as: percent of death in infected sample minus percent of baseline death in noninfected sample. For terminal deoxynucleotidyl transferase-mediated dUTP nick-end labeling (TUNEL) assays, $2 \times 10^{6} \mathrm{CD} 4 \mathrm{~T}$ cells were incubated with $10^{6} 293 \mathrm{~T}$ cells at $37^{\circ} \mathrm{C}$ for 24 hours. Cells were treated with terminal deoxynucleotidyl transferaseand strained with FITC-labeled UTP according to manufacturer's instructions (Coulter Beckman Electronics Ltd., M iami, Florida, USA). In these experiments, Iymphocytes were isolated by sel ectively gating on CD $4^{+}$ cells in which cell death was subsequently analyzed.

To determine the expression of CD 4 and chemokine receptor, $10^{6} \mathrm{~T}$ cells were stained with anti-CXCR4 (12G5)-FITC (Becton Dickinson Immunocytometry Systems), anti-CCR5 (2D 7)-FITC (PharM ingen, San Diego, California, USA), anti-CD 4-phycoerythrin (anti-CD 4-PE) (Caltag Laboratories Inc., Burlingame, California, USA), or mouse isotype controls (Becton Dickinson Immunocytometry) as previously described (6). Flow cytometry was performed using a FACScan (Becton Dickinson Immunocytometry Systems), and analysis was doneusing CellQ uest software. Laser-scanning confocal microscopy was performed using a Zeiss LSM -510 (Carl Zeiss I nc., Thornwood, N ew York, USA). Images were saved at eight bits per channel and were analyzed for fluorescent interacting using a computer analysis package ANALYZE (Mayo Foundation, Rochester, M innesota, USA).

HIV infection. Five-day-old phytohemagglutinin (PHA) ( $5 \mu \mathrm{g} / \mathrm{ml}$; Roche M olecular Biochemicals) and $200 \mathrm{IU}$ of IL-2 (Chiron Therapeutics, Emeryville, California, USA) treated $5 \times 10^{6} \mathrm{CD} 4 \mathrm{~T}$ cells were infected with the X4 HIV strain (LavBru) and the R5 HIV strain ${ }_{(\mathrm{R}-\mathrm{FL})}$ (NIH AIDS Research Reference Program) (360 ng of p24) or mock infected. Cells were thereafter passed every 3 days at a concentration of $10^{6} \mathrm{cell} / \mathrm{s} / \mathrm{ml}$. For some experiments (seeFigure 5b), 4- to 6-day-old HIV-infected CD 4 T cells werewashed and treated with $5 \mu \mathrm{M}$ of AZT for 6 hours, followed or not followed by the addition of $20 \mu \mathrm{M}$ of thevarious caspaseinhibitors. Eighteen hours later cells werefixed and permeabilized (Fix and Perm; Caltag Laboratories) and stained for intracytoplasmic HIV p24 using anti-p24-FITC Ab's (Coulter Beckman Electronics Ltd.). Flow cytometry was performed using FACScan, and analysis was done using CellQ uest software. Statistical analysis. Experiments from every figure were performed in duplicate and repeated at least three times. All measurements are presented as means and SD's with statistical comparisons made between conditions using theStudent'st test for paired observations.

\section{Results}

Individual activation of CCR 5 and CD 4 in uninfected CD 4 T cells leads to a caspase 8-dependent death. To investigate how HIVenv/chemokine coreceptor interactions may mediateCD 4 T-cell death, wefirst investigated whether 
R5env mediates death of uninfected T cells. Purified resting CD 4 T lymphocytes were coincubated with 293T cells expressing R5env or empty vector. R5env, as compared with empty vector, caused CD 4 T-cell death, which was blocked by the pancaspase inhibitor Z-VAD (Figure 1a). Because R5env interacts with both CD 4 and CCR5 receptors, the function of each receptor in mediating R5env-triggered death was investigated individually. To study the R5env-triggered death by CCR5, 293T cells expressing R5env or empty vector were preincubated with solubleCD 4 (SCD 4). This protein blocks the interaction of HIVenv with membrane CD 4 and also causes a conformational change that enhances its binding to the chemokine receptor (19-22). When 293T cells expressing R5env were treated with different concentrations of SCD 4, a caspase-dependent CD 4 Tcell death continued to be observed (Figure la), suggesting that CCR5 mediates a caspase-dependent death of uninfected CD 4 T cells. To identify the caspase mediating the R5env/CCR5-induced death, CD4 T cells werepretreated with caspase 8 (Z-IETD) or caspase 9 (Z-LEHD) inhibitors immediately before their coincubation with R5env-expressing 293T cells (preincubated with SCD 4). The fact that Z-IETD, but not ZLEHD, reversed the R5env/CCR5-induced death (Figure la, right panel) suggests that caspase 8, but not
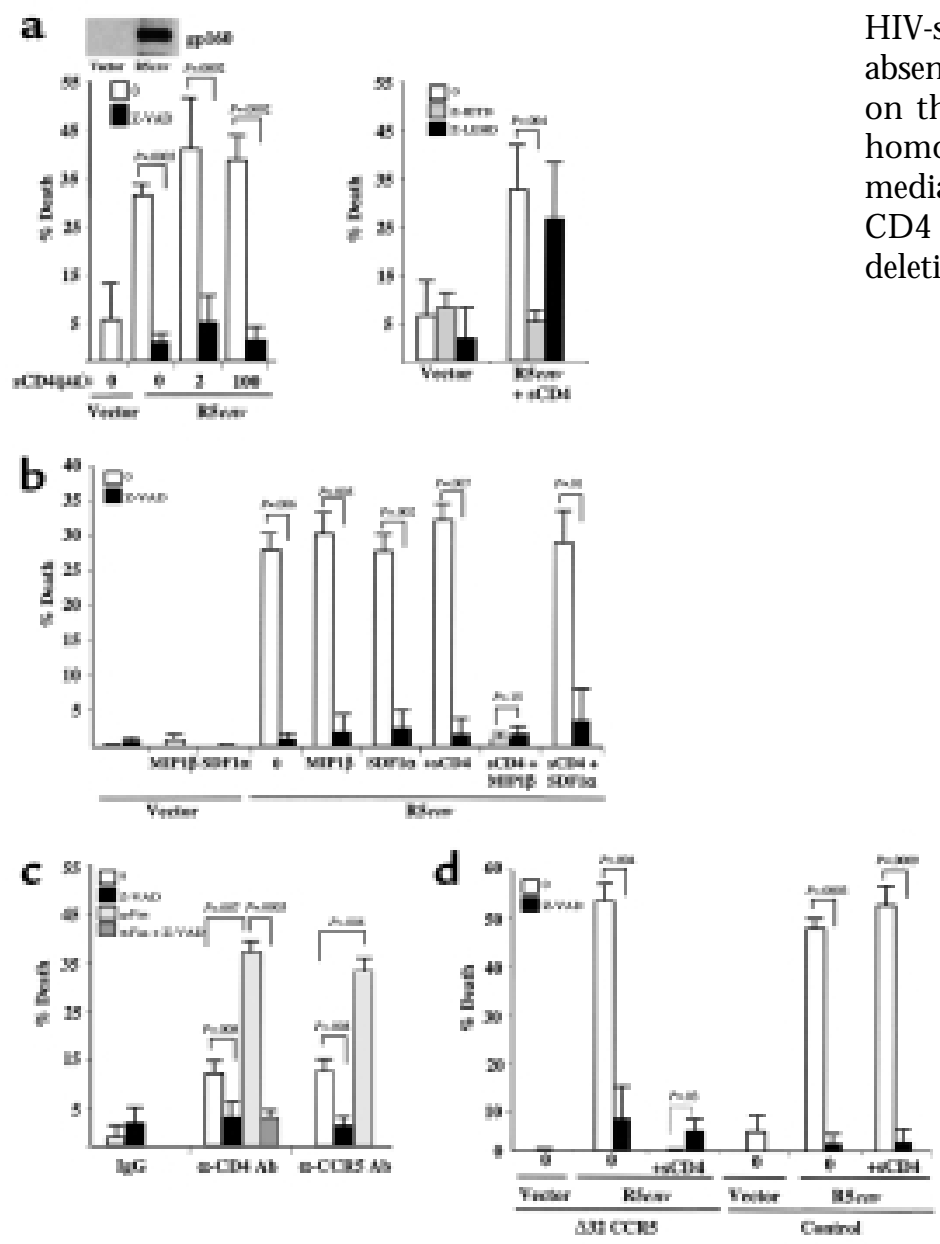

9, is involved in the R5env/CCR5-induced T-cell death.

To confirm that CCR5 mediates R5env-triggered death of CD 4 T cells, the cells were treated with the natural ligand of CCR 5 (MIP1 $\beta$ ) and, as control, with the natural ligand of CXCR4 (SDF $1 \alpha$ ) before their coculture with R5env-expressing 293T cells in the presence or absence of SCD 4. As shown in Figure 1b, MIP1 $\beta$, but not SDF1 $\alpha$, abrogated the caspasedependent death triggered by R5env in the presence of SCD 4. Both MIP1 $\beta$ and SDF $1 \alpha$ treatment completely abrogated the expression of CCR5 and CXCR4 in these cells, respectively, as determined by using flowcytometric analysis (not shown) and did not cause death (Figure $2 b$ ).

When these experiments are performed in the absence of SCD 4, thus allowing for the interaction of R5env with both CD 4 and CCR5, neither MIP1 $\beta$ nor SDF $1 \alpha$ blocked the R5env-mediated caspase-dependent death. This suggests that both CD 4 and CCR 5 individually and independently mediate the caspasedependent death when activated by R5env. Additional experiments confirmed that the CD 4-mediated death also requires caspase 8, but not caspase 9 (data not shown and Figure 2d). The role of CCR 5 and CD 4 in individually mediating caspase-dependent death was further confirmed using cross-linking Ab's against each receptor (Figure 1c).

The CCR 532 deletion present in CD $4 \mathrm{~T}$ cells from HIV-seropositive individuals is associated with low or absent HIV replication and CD 4 T-cell depletion. Based on this, we argued that CD 4 T cells carrying such homozygous deletion would be refractory to R5envmediated death by CCR 5 but not by CD 4 . To test this, CD $4 \mathrm{~T}$ cells from a healthy donor with a CCR $5 \Delta 32$ deletion werecompared with thosefrom a control indi-

\begin{abstract}
Figure 1
CCR5 activation induces a caspase-dependent death of CD 4 T cells. (a) CD4 T cells were incubated with 293T cells transfected with empty vector or 293 T cells expressing the

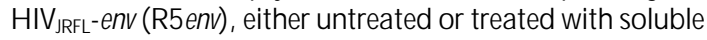
CD4 at different concentrations (SCD4). 293T cells were lysed and blotted by duo, tropic anti-gp160 to verify envelope expression (left panel). CD4 T cells were left untreated, pretreated with Z-IETD (a caspase 8 inhibitor) or Z-LHED (a caspase 9 inhibitor) before incubation with 293T cells expressing empty vector or R5env (right panel). (b) CD4 T cells treated or not treated with Z-VAD, SDF1 $\alpha$, or MIP1 $\beta$ were incubated with 293T cells expressing R5env or empty vector pretreated or not pretreated with $S C D 4$, respectively. (c) CD4 T cells were treated with IgG-matched isotypecontrol, anti-CD4 Leu-3a ( $\alpha$ CD4 Ab), or anti-CCR5 MAB183 ( $\alpha C C R 5 A b)$, followed by their cross-linking with goat antimouse Ab's. Each point was assessed for caspase-dependent death (Z-VAD) as well as for Fas susceptibility ( $\alpha$ Fas). (d) CD $4 \mathrm{~T}$ cells from a healthy donor with the CCR5 $\Delta 32$ mutation or from a control donor lacking the $\triangle 32$ CCR5 deletion were pretreated or not pretreated with Z-VAD and coincubated with $293 \mathrm{~T}$ cells expressing R5env or empty vector, pretreated or not pretreated with SCD4.
\end{abstract}


vidual lacking such deletion with regard to the ability of R5env to mediate CD 4 T-cell death by CCR5. As shown in Figure 1d, CCR5 $\triangle 32$ CD 4 T cells werekilled by R5env in the absence of SCD 4. H owever, preincubation of R5env-expressing 293T cells with SCD 4 completely abrogated CD 4 T-cell death from the $\triangle 32$ CCR5 donor, but not from the control individual. Taken together these data indicatethat CCR5 is functional in mediating the R5env-triggered, caspase 8-dependent, CD 4 T-cell death independently of CD 4 .

CXCR 4 activation causes a caspaseindependent death of uninfected CD $4 \mathrm{~T}$ cells. Based on our observation that CCR5 can mediate R5env death, wequestioned whether
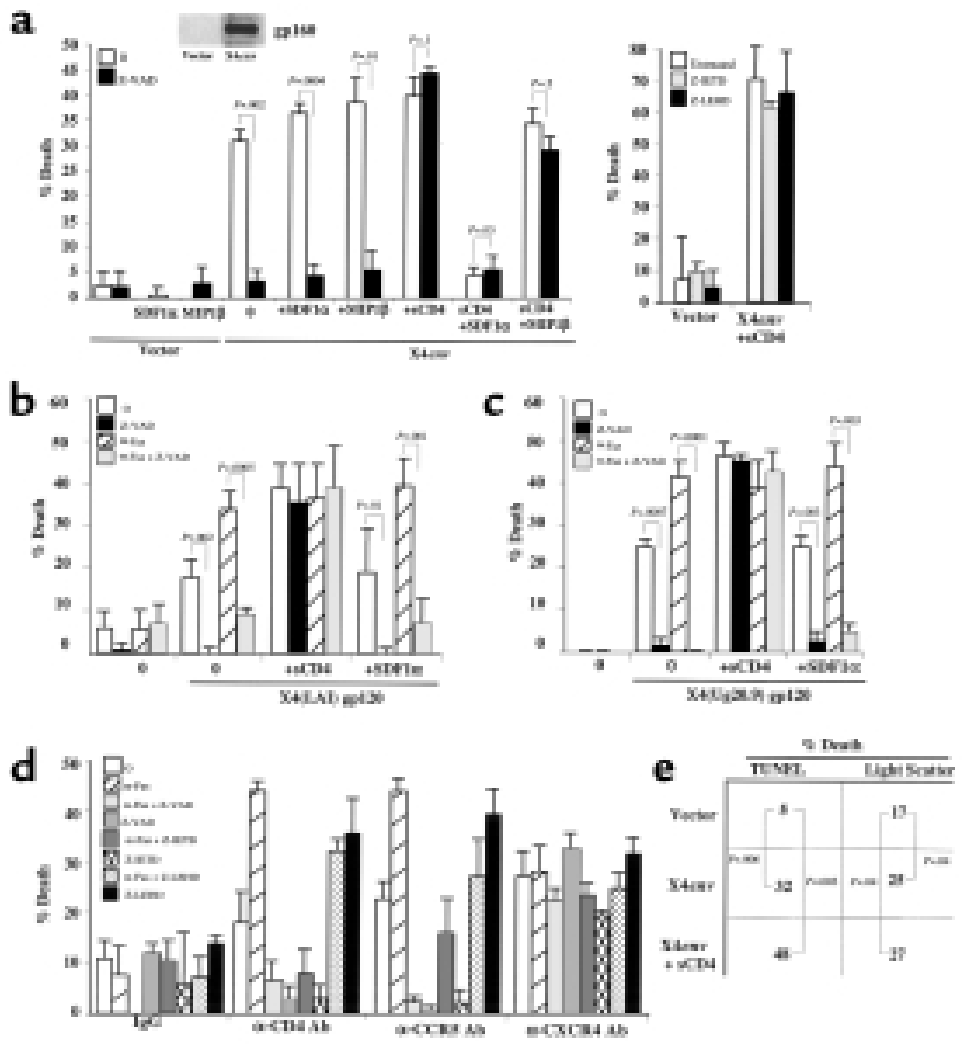

Figure 2

CXCR4 activation induces a caspase-independent death of CD4 T cells. (a) CD4 T cells treated or not pretreated with Z-VAD, SDF1 $\alpha$, or M IP1 $\beta$ were incubated with 293T cells transfected with empty vector (vector) or $\mathrm{HIV}_{\mathrm{HXB2}}$-env (X4env), and untreated or pretreated soluble CD4 (SCD4) (left panel). CD4 T cells were untreated or pretreated with Z-IETD (a caspase 8 inhibitor) or Z-LEHD (a caspase 9 inhibitor) before incubation with 293T cells expressing empty vector or X4env (right panel). (b) CD4 T cells were untreated or treated with soluble gp120 (X4 gp120) alone or in combination with soluble CD4 (SCD4). Some cells were pretreated with SDF1 $\alpha$ to block CXCR4 receptors. Caspase-dependent death was analyzed using Z-VAD (Z-VAD), and Fas susceptibility was analyzed using treatment with anti-Fas Ab's ( $\alpha$ Fas). (c) CD 4 T cells were preincubated or not preincubated with SDF1 $\alpha$ and treated or not treated with soluble primary (92Ug20.9) X4 gp120 that was preincubated or not preincubated with sCD4. (d) CD 4 T cells were cross-linked with anti-CD 4 Leu-3a ( $\alpha$ CD4 Ab), anti-CCR5 MAB 183 ( $\alpha C C R 5 A b)$, anti-CXCR4 12G5 ( $\alpha$ CXCR4 Ab), or matched isotypecontrol (IgG) and assessed for death and/or Fas susceptibility as in $\mathbf{b}$ in the presence or absence of the pancaspase inhibitor (Z-VAD), caspase 8 inhibitor (Z-IETD), and caspase 9 inhibitor (Z-LEHD). (e) CD4 T cells were preincubated with 293T expressing or not expressing X4env preincubated or not preincubated with SCD4. Cell death was analyzed using changes in the light scatter or by TUNEL analysis using FACS. the other HIV coreceptor (CXCR4) could exert a simicaspase-dependent manner (Figure 2a, left panel). Because the X4env interacts with both CD4 and XCR4, we further investigated the individual role of receptor. Surprisingly, when X4env-expressing incurred in CD $4 \mathrm{~T}$ cells was caspase independent.

To confirm that CXCR4 can mediate a caspase-independent death when activated by X4env, CD 4 T cells ncubated with the CXCR4 ligand (SDF1 $\alpha$ ) and, as control, with the CCR5 ligand (MIP1ß) (23-25) beforetheir coculturewith 293T cells expressing X 4env in the presence or absence of SCD4. SDF1 $1 \alpha$, but not MIP1 $\beta$, blocked theX4env-triggered (preincubated with SCD4) death of CD 4 T cells (Figure 2a). However, in the absence of SCD 4, neither SDF $1 \alpha$ nor MIP $1 \beta$ inhibited the death of $C D 4 T$ cells triggered by $X 4$ env, once more highlighting that $\mathrm{CD} 4$ alonecan trigger a caspase-dependent death when activated in isolation by either R5env or X4env. As expected, neither the caspase 8 nor caspase 9 inhibitor blocked the $X 4$ env/SCD 4 death of CD 4 T cells (Figure $2 a$, right panel), and SDF $1 \alpha$ or MIP1 $\beta$ treatment did not cause CD 4 T-cell death (Figure 2a) (26). Therefore, $X 4$ env can trigger a caspase dependent death when interacting with CD 4 but a caspase-independent one when interacting with CXCR4. This is in striking contrast with the R5env-mediated death, which is caspase dependent regardless of its interaction with CD 4 or CCR5.

Theability of X 4env/CXCR4 interactions to cause a caspase-independent CD $4 \mathrm{~T}$-cell death was further confirmed by activating CXCR4 through other ligands, such as solubleX4env and anti-CXCR4 Ab's. As shown in Figure 2, b and c, two different soluble X4 gp120 (a laboratory-adapted strain, or LAI, and a patient-derived primary X 4 HIV strain, 92U g20.9) killed CD 4 T cells in a caspase-independent manner in the presence of SCD 4; an effect that was reversed in the presence of SDFl $\alpha$. Likewise, when CD 4 T cells were incubated with antiCXCR 4 cross-linked Ab's, a caspase-independent death was observed (Figure 2d). This contrasts with the caspase 8-dependent death that ensues when CD $4 \mathrm{~T}$ cells are activated by CD 4 or CCR5 (Figure 2d).

Both the CD4- and CXCR4-mediated CD 4T-cell death were also evident by using TUNEL and changes in FSC were evident by using flow cytometry, suggesting both 
CD4- and CXCR4-triggered T-cell death are a form of an apoptotic process, even though it is mediated by different molecular mechanisms.

Therelationship between CXCR4 and CD 4 expression influences themechanism of uninfected CD $4 \mathrm{~T}$-cell death. Although $X 4$ env binds to both CD 4 and CXCR4, only a CD 4 caspase-dependent death is observed when $X 4$ env is incubated with primary resting CD $4 \mathrm{~T}$ cells, suggesting that the CD 4 signal overrides the CXCR4 signal. This could bedue to cross-talk between both receptors resulting in, possibly, the desensitization of CXCR4 signaling by CD 4 $(27,28)$ and/or to the relative expression level of each receptor. Recent studies in HIV-infected patients demonstrate that lymphocytes in the periphery and lymphoid tissue havea decreased CD 4/CXCR4 ratio resulting from higher expression of CXCR4 when compared with noninfected patients (29). This prompted us to investigate whether upregulation of CXCR4 expression in primary CD $4 \mathrm{~T}$ cells would favor a X4env-mediated, caspase-independent death. For this purpose we used IL-4 to increase thesurfaceexpression of CXCR4 on T Iymphocytes, and high levels of this cytokine are present in patients with latestageHIV infection when a predominance of X4 HIV strains is noted $(30,31)$. In the absence of IL-4, X4 gp120 triggered a caspase-dependent cell death and further rendered thecell susceptibleto Fas-mediated apoptosis (Figure 3a). However, in the IL-4 treated CD 4 T cells, X4 gp120 induced a CXCR4, caspase-independent death (Figure 3a). Addition of SDF $1 \alpha$ to IL-4-treated CD 4 T cells reverted the $X 4$ gp120-mediated death to a CD 4 caspase-dependent death (Figure $3 a$ ). Therefore, X4env favors the CXCR4, caspase-independent mode of death when CXCR4 expression is increased relative to CD 4.

Chemokine receptors are constantly cycling to the cell surface; therefore, the percentage of CXCR4-expressing cells at any timepoint may underestimatetheoverall level of surface expression that is present during a 24-hour period in which the CD $4 \mathrm{~T}$ cells are exposed to the different ligands. This could account for potential differences in the degree of death observed with respect to the chemokinereceptor expression (32, 33). Using FACS and confocal-microscopy analysis, we demonstrated that IL4 resulted in CXCR 4 surface expression upregulation per individual cell, which was suppressed by SD F $1 \alpha$, while not modifying CD 4 surfaceexpression (Figure $3 b$ ). While we can not ruleout the possibility that IL-4 may directly enhance CXCR4 activity or interfere with the potential desensitization of CXCR4 by CD 4, weconfirmed that IL4-treated or -untreated CD $4 \mathrm{~T}$ cells remained equally susceptibleto Fas-mediated apoptosis after cross-linking of CD 4 with anti-CD 4 Ab's followed or not followed by anti-Fas Ab treatment (data not shown).

X4env/CXCR4 interaction causes a direct caspaseindependent death of $C D 8 T$ cells. The observation that $X 4 \mathrm{env}$, but not R5env, triggered caspase-independent CD $4 \mathrm{~T}$ lymphocyte death by CXCR 4 raised the possibility that CD $8 \mathrm{~T}$ cells may also be direct targets for X4env. This scenario is clinically relevant because CD $8 T$ cells, which are important in the control of HIV infection, areknown to beprogressively depleted in thelatestages of HIV infection, especially when X4 HIV strains predominate $(15,16)$. Purified resting CD 4 or CD 8 T cells from the samedonor wereseparately coincubated with soluble recombinant X4 (LAI) gp120. As expected, X4 gp120 triggered death of resting, non-IL-4-treated, CD 4 T cells by a caspase-dependent mechanism and further sensitized CD 4 T cells to Fas-dependent apoptosis (Figure 4a). H owever, X4 gp120 triggered CD 8 Tcell death that was not blocked by caspase inhibitors and did not sensitize the CD8 T cell to Fas-mediated apoptosis (Figure 4a). CD 8 T-cell death by apoptosis was again confirmed by changes in FSC/SSC by the use of flow cytometry (seeFigure 4a legend). In this experiment, the surface expression of CXCR4 was similar in both CD 4 and CD 8 T cells (17\%and 18\%, respectively).

To demonstrate that the $\mathrm{X} 4 \mathrm{gp} 120$-mediated death of primary CD $8 \mathrm{~T}$ cells was triggered by CXCR4, purified CD 8 T cells were treated or not treated with SDF $1 \alpha$ before the addition of $X 4 \mathrm{gp} 120$. As shown in Figure $4 \mathrm{~b}$, the caspase-independent death of CD $8 \mathrm{~T}$ cells triggered by $X 4$ gp 120 was significantly decreased in the presence of SDF1 $\alpha$, which concurrently decreased CXCR 4 expression in the same cells (Figure $4 \mathrm{~b}$, inset). The role of X4env/CXCR 4 interactions in mediating CD8 T-cell death through caspase-inde-

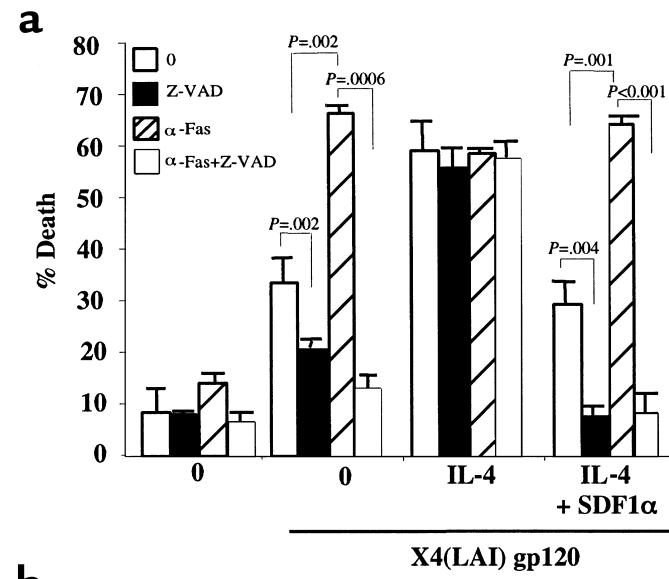

b

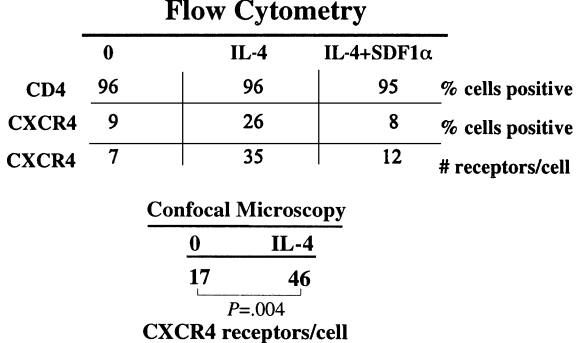

Figure 3

CXCR4/CD 4 receptor ratio determines the mode of CD 4 T-cell death. (a) Untreated, IL-4 alone, or IL-4- and SDF1 $\alpha$-treated CD 4 T cells were incubated or not incubated (0) with soluble X4 gp120. Cells were assessed for Fas ( $\alpha$ Fas) susceptibility and caspase-dependent death (Z-VAD). (b) CD4 T cells from a were simultaneously stained for CD4 and CXCR4 and analyzed using flow cytometry. CD4 T cells stained for CXCR4 were also analyzed using confocal microscopy. 
pendent mechanisms was again confirmed using crosslinked anti-CXCR4 Ab's (Figure 4c).

Wenext investigated whether CD 8 T-cell death could result from interactions with cells expressing membrane-bound X 4 gp160 and whether R5env played a role in killing CD 8T cells. For this, X4env- or R5env-expressing 293T cells were incubated with either purified CD 4 or CD8 T cells from the same donor in the presence or absence of caspaseinhibitors. While R5env cau sed a caspase-dependent cell death of CD 4 T cells, it did not induce death of purified CD $8 \mathrm{~T}$ cells (Figure 4d). Interestingly, X4env-expressing cells caused a caspasedependent death of resting, untreated CD $4 T$ cells and a caspase-independent death of CD 8T cells (Figure4d). The expression of CCR5 in this experiment was higher in CD 8 than CD 4 T cells ( $15 \%$ and $5 \%$, respectively), while CXCR4 expression was the same (15\% and $14 \%$, respectively). As seen in Figure 3 for CXCR4, the expression level of CCR5 may underestimate the real expression present during the duration of theexperiment due to therapid cycling of thesereceptors to thecell surface. Therefore, the inability of R5env to cause CD 8 T-cell

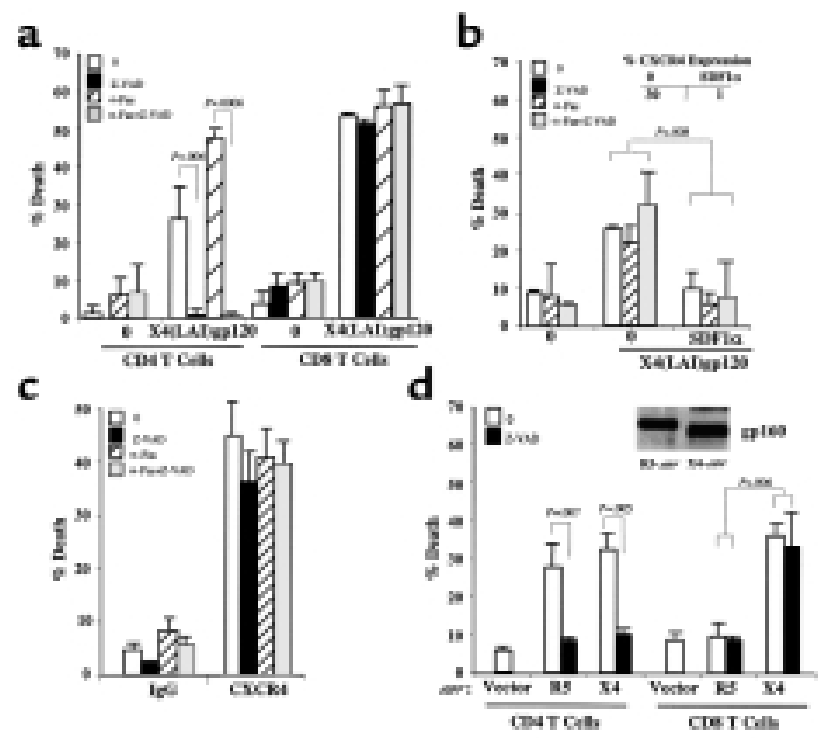

Figure 4

CD8 T cells die by HIVenv-CXCR4 infection. (a) Purified CD4 and CD 8 T cells were untreated (0) or treated with soluble X4 (LAI) gp120 and assessed for Fas susceptibility ( $\alpha$ Fas) or caspase-dependent death (ZVAD). Ten percent of untreated cells, $23 \%$ of cells treated with $X 4$ gp120, and $24 \%$ of cells treated with Z-VAD and X4 gp120 ( $<<0.006)$ demonstrated changes in FSC compatible with apoptosis in flow cytometry. (b) CD8 T cells were untreated (0) or treated with SDF1 $\alpha$ before incubating with X4 (LAI) gp120 and analyzed for caspasedependent death (Z-VAD) or susceptibility to Fas-mediated apoptosis ( $\alpha$ Fas). CD8 T cells were simultaneously analyzed using flow cytometry for CXCR4 expression with and without SDF1 $\alpha$ treatment (inset). (c) CD8 T cells were cross-linked with anti-CXCR4 12G5 Ab ( $\alpha$ CXCR4 Ab) or matched isotype control (IgG) and assessed for Fas susceptibility ( $\alpha$ Fas) or caspase-dependent death (Z-VAD). (d) CD4 and CD8 T cells were mixed with $293 \mathrm{~T}$ cells expressing empty vector (vector), $\mathrm{HIV}_{\text {JRFL-env }}$

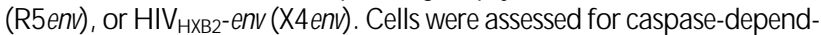
ent death (Z-VAD). Transfected 293T cells were lysed and blotted by duotropic anti-gp160 Ab to verify envelope expression (inset). death was not dueto lack of CCR5 expression.

In conclusion, these findings highlight the different molecular mechanisms whereby death of uninfected primary $T$ cells can be triggered by HIVenv through its specific interaction with chemokine receptors. Furthermore, our results confirm that both soluble and membrane-bound env mediate the same cell-death mechanisms and that $X 4$, but not R5env, can directly kill CD $8 \mathrm{~T}$ cells by CXCR4.

HIV-infected CD 4 T cells die via chemokine receptor-mediated mechanisms. Direct killing of HIV-infected CD 4 T cells has been proposed by some groups as a major cause of CD 4T-cell depletion in HIV-infected patients. This death is thought to be mediated by caspase-inde pendent mechanisms $(9,10)$. Interestingly, such conclusions have been determined through the use of $X 4$ HIV strains. Our observation that HIVenv can signal different modes of death to uninfected $T$ cells, depending upon which chemokine receptor is activated, led us to question whether the HIV coreceptors would also mediate the so-called direct death of the HIV-infected cell. If so, we would predict that R5 HIV strains would kill CD 4 T cells via caspase-dependent mechanisms, an effect that would be reversed in the presence of the CCR5 ligand, MIP1ß. On the contrary, we would predict that X4 HIV strains could kill via caspase-independent mechanisms and that the death would be blocked by SDF1 $\alpha$, if X4 HIV-mediated death is dependent upon theisolated interaction of $X 4$ env with CXCR4. Such observations would bridge the two schools of thought arguing for direct or indirect death as being caused by separate molecular mechanisms.

CD 4 T cells were infected in parallel with R5 $\mathrm{HIV}_{\mathrm{IIR}}$ $\mathrm{FL}$ ) or $\mathrm{X} 4 \mathrm{HIV}_{\text {(Lav-Bru) }}$ strains and treated or not treated with the pancaspaseinhibitor Z-VAD immediately after infection. We observed that CD $4 \mathrm{~T}$ cells infected with R5 HIV died via a caspase-dependent mechanism, similar to the mechanism of death observed in uninfected cells after R5env interactions with CD 4 and CCR5. In contrast, CD $4 \mathrm{~T}$ cells infected with X4-HIV died via a caspase-independent mechanism (Figure 5a), resembling the X4env/CXCR4-mediated death of uninfected IL-4-treated cells. HIV p24 values were higher in the culture supernatants of the R5 HIV-infected ZVAD-treated samples, than the R5 HIV-infected non-Z-VAD-treated samples (Figure 5a legend), indirectly reflecting the higher viability of infected cells and henceresulting in overall higher viral load. The known downregulation of CD 4 and potential upregulation of CXCR4 during HIV infection (29, 34-36) may shift the CD 4/CXCR4 ratio, thus favoring a predominant interaction between $X 4$ env and CXCR4, ultimately resulting in a caspase-independent death. This would be similar to the pattern observed with IL-4 treatment in the uninfected CD 4 T cells (Figure 3). The direct HIVmediated cell death was further confirmed by FACS analysis (see Figure 5a legend). In addition, this experiment was repeated using two other R5 (SF 162) and X4 (IIIB) HIV strains, yielding the same results. 
To further characterize the specific caspase involved in the R5env-mediated death, additional experiments, similar to those described in Figure 5a, were performed, except that the HIV-infected cultures were incubated in the presence of the sel ective caspase 8 inhibitor (Z-IETD) or the caspase 9 inhibitor (ZLEHD). As shown in Figure 5b, only Z-IETD inhibited the R5 HIV-mediated death. As expected from results shown in Figure 5a, X4 HIV-induced CD 4 Tcell death was not blocked by either Z-IETD or ZLEHD. This further suggests that the death of uninfected and HIV-infected cells by R5env and R5 HIV, respectively, is mediated by similar mechanisms.

To address formally whether it is the HIVenv interaction with the corresponding chemokine coreceptor that ultimately triggers death of theinfected cells, we investigated whether blocking such interaction would result in decreased HIV-mediated death. Such observation would strongly argue for the hypothesis that it is the HIVenv viral protein that is ultimately responsible for the death of both uninfected and HIV-infected CD $4 T$ cells. To test this hypothesis, we used thenatural ligand of each chemokine receptor (MIP1 $\beta$ for CCR5 and SDFl $\alpha$ for CCR4) as inhibitors of the HIVenv/chemokinereceptor interaction. This approach was proven to be feasible for the study of uninfected CD 4 T cells (Figure $1 b$ and $2 b$ ). To avoid the interference of the corresponding chemokine with new cycles of infection, HIV-infected CD 4 T cells werefirst treated with thereversetranscriptase inhibitor, AZT. As shown in Figure 5c, the death observed in R5 HIV-infected, AZT-treated cells was not blocked by SDF $1 \alpha$ but was significantly reduced by MIP1 $\beta$. Conversely, the death observed in X4 HIV, AZT-treated cells was significantly reduced by SDF $1 \alpha$, but not by MIP1 $\beta$. The number of CD 4 T cells expressing intracytoplasmic p24 (Figure 5d and data not shown) was not different in the AZT-treated cells incubated or not incubated with the various chemokines, suggesting that the chemokine effect was exerted by interfering with the env/chemokine receptor interaction rather than by reducing HIV infection.

To investigatewhether env expressed in theHIV-infected CD $4 \mathrm{~T}$ cells mediates the death of both the infected and uninfected CD $4 \mathrm{~T}$ cells present in the HIV-infected culture, primary CD 4 T-cell blasts were infected with $\mathrm{X} 4_{(\text {Lav-Bru }}$ or R5 (UR-FL) $\mathrm{HIV}$ strains. On days 4-6 after infection thecells weretreated with AZT to block any further infection. This was donein a similar manner as for Figure $5 \mathrm{c}$ when $15-20 \%$ of cells were determined to beHIV infected by intracytoplasmic p24 staining, becauseinfection rates beyond these levels resulted in very rapid cell death that precluded adequate analysis using flow cytometry. After AZT treatment, half of the culture was treated with the pancaspase inhibitor Z-VAD, and after 18 hours, cells were analyzed for intracytoplasmic p24 and apoptosis using flow cytometry. Results from these experimentsindicated that $\mathrm{CD} 4 \mathrm{~T}$-cell death was present in both the uninfected (p24-) and HIV-infected (p24+) cells (Figure5d). As expected, the R5 HIV-mediated death of both infected and uninfected CD 4 T cells was mediated via a caspase-dependent mechanism, whereas the $X 4$ HIV-mediated death was not. Thehigher percentage of

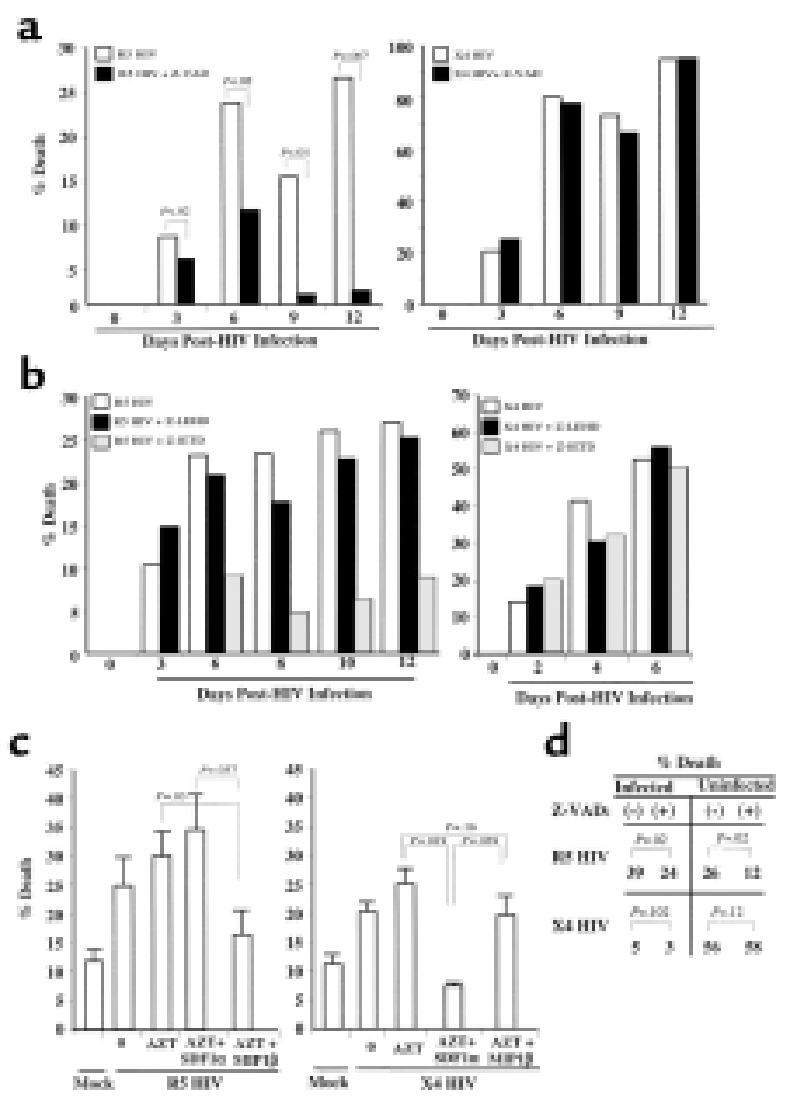

Figure 5

Infected CD4 T-cell blasts die by chemokine receptor-dependent mechanisms. (a) PHA and IL-2-stimulated cells were infected with X4 HIV (Lav- $_{\text {- }}$ $\mathrm{Bru}$, or R5 HIV $\mathrm{H}_{\text {(JFL), }}$ or mock (NI) virus. Cells were counted every 2-3 days and analyzed for cell death or by using flow cytometry changes in FSC consistent with apoptosis and for PI cell cycle. On day 6 of infection the percentage of cells infected with the R5 HIV strain shown to be apoptotic by using PI staining were $25 \%$ (- Z-VAD) and $15 \%$ (+Z-VAD) or by FSC were $29 \%(-Z-V A D)$ and $19 \%$ (+Z-VAD). On day 9 of infection the percentage of cells infected with X4 HIV shown to be apoptotic by PI staining were $71 \%$ (- Z-VAD) and 69\%(+Z-VAD) or by FSC were $68 \%(-$ Z-VAD) and 64\% (+Z-VAD). p24 values from the supernatants were: R5, 293(+)Z-VAD and 178(-)Z-VAD ( $<<0.1)$; X4, 41(+)Z-VAD and 40(-) ZVAD. $S D=<10 \%$ at each data point. (b) The procedure was the sameas in a, except cells were incubated in the presence or absence of caspase 8 inhibitor (Z-IETD) or caspase 9 inhibitor (Z-LEHD). (c) PHA and IL-2 stimulated and infected with R5 HIV (JR-FL) $_{\text {, or X4 HIV }}$ (Lav-Bru), or mock infected were treated with AZT for 6 hours on day 3 of X4 HIV infection and on day 6 of R5 HIV infection. Thereafter, cells were extensively washed and incubated in the presence or absence of SDF1 $\alpha$ or MIP1 $\beta$, and cell death was analyzed 24 hours later. (d) Chemokine-receptor activation mediates death of directly HIV-infected and -uninfected CD4 T cells. PHA and IL-2-stimulated cells were infected with R5 HIV $\mathrm{V}_{(\mathrm{JR}-\mathrm{FL})}$, or X4 HIV (Lav-Bru), or mock infected (NI). O n day 4 for X4 HIV infection and day 6 for R5 HIV infection, cells were treated with AZT, and half the population was treated with Z-VAD; 18 hours later cells were stained for intracytoplasmic p24. The percentage of p24+cells and apoptotic cells, as determined changes by FSC, were analyzed by using flow cytometry. The percentage of p24+ in the R5 HIV infection of T cells was $11 \%$ and $15 \%$ in the X4 HIV infection ( + ). SD $=<10 \%$ at each data point. 
death observed in the uninfected versus infected cells within the X4 HIV-infected cultures is a function of the experimental design, becausein later, postinfection times a higher degree of death is observed in the $124^{+}$cells. Therefore, HIVenv activation of thechemokinereceptors mediates the death of both infected as well as uninfected CD 4 T cells present within thesameHIV infected culture.

Taken together, these results indicate that the interaction of the HIVenv with the corresponding chemokine coreceptor mandates the molecular mechanism of T-cell death in both the uninfected bystander and HIV-infected T cells.

\section{Discussion}

N early a decade ago, HIVenv was proposed and confirmed as an HIV protein responsible for the direct killing of HIV-infected CD4 T cells (4). Soon after, it became clear that the env, either in a soluble or membrane-bound form, could also mediate death of uninfected bystander T cells (2-6). The recent identification of the HIV coreceptors has presented the opportunity to study their role in the HIVenv-mediated death. Our results have highlighted that the specific HIVenv-coreceptor interaction dictates the molecular mechanisms of T-cell death. M oreover, wehave found a striking similarity between how HIV-infected cells and uninfected $T$ cells die. Specifically, CCR 5 activation induced a caspase-dependent death of both infected and uninfected CD 4 T cells, and CXCR4 activation by X4env caused a caspase-independent death of both infected and uninfected CD 4 T cells and uninfected CD 8 T cells. This may explain the conclusions drawn by several studies arguing for a lack of caspase-mediated death of HIVinfected cells when X4 HIV strains were used. The bifunctional role of $X 4$ env in causing a caspasedependent death when interacting with the CD 4 receptor, but a caspase-independent death when interacting with the CXCR4 receptor, highlights the separateroles of these two receptors in mediating lymphocyte death. Furthermore, these results are consistent with recent observations arguing that X 4 env is capable of mediating caspase-dependent death (37). Also, our results could explain the differences in rate and location of CD 4 T-cell depletion by R5 or X4 SHIV-infected macaques, reported by $\mathrm{H}$ arouse et al. (17).

The peripheral T-cell depletion characteristic of HIV infection is thought to be due, at least in part, to death of uninfected T cells. The death of these uninfected T cells has been shown to occur in lymphoid tissue from HIV-infected patients when contacted by an HIVinfected cell (5). Our results suggest this may be explained by membrane-env-triggered death. In addition, soluble gp120 produced within the infected lymphoid tissue could also directly kill or sensitize the T cell to subsequent death. We have demonstrated that CD 4 T-cell death occurs in both the HIV-infected and uninfected cells within the HIV-infected culture. Independently of the exact proportion of infected versus uninfected cell dying, our results clearly support that env-mediated killing by the corresponding chemokine receptor mandates the form of death for both infected and uninfected cells present in the HIV-infected culture. From previous studies that calculated theamount of viral proteins present in lymphoid tissue, concentrations of solublegp120 ranging between 120 and 960 $\mathrm{ng} / \mathrm{mL}$ may exist in the lymph nodes of HIV-infected individuals (38-40). In fact, we performed doseresponsestudies of solublegp 120 and found that concentrations as low as $500 \mathrm{ng} / \mathrm{mL}$ were sufficient to mediate significant T-cell death $(P<0.006)$.

Our results also raise the possibility that CD $8 \mathrm{~T}$ cells may be depleted in vivo if enough $X 4$ env-expressing infected T cells or solubleX4env arepresent within lymphoid tissue. Previous studies have demonstrated that CD8T cells, in addition to CD 4 T cells, undergo apoptosis in lymphoid tissuefrom HIV-infected patients (5, 41,42 ). The conclusion of such studies has been generally that CD 8 T-cell death is secondary to their aberrant activation. An alternative explanation is that they are actively dying by X4env/CXCR4 interactions. Supportive evidencefor this line of reasoning could result from determining whether R5 HIV-infected lymphoid tissue does not contain CD 8 T-cell death, whereas X4 HIV does. However, our results contradict, in part, other studies that report that $X 4$ env indirectly triggers death of CD 8 T cells by macrophage production of TNF (16). Using highly purified CD $8 T$ cells in which every effort was made to limit monocyte contamination, we have shown that caspase inhibitors did not block X4env/CXCR4-triggered CD 4 T-cell death, arguing against a rolefor TNF-mediated death in our studies, and hence of any effector monocyte contamination, because TN F-mediated death is caspase dependent. We believe that definitive proof that X4env mediates CD 8 T-cell death will require analysis of lymphoid tissue of X4 HIV-infected patients because in vitro cultures of primary $T$ cells, or even ex vivo infection of lymphoid tissue, may not ultimately reflect the true in vivo situation. This is important when considering AIDS pathogenesis, because CD $8 \mathrm{~T}$ cells are key in the control of this chronic viral infection. The depletion and increased uncompensated turnover of CD $8 \mathrm{~T}$ cells observed in thelatestages of HIV infection, when more X4 strains may be present, will hinder the immune clearance of the virus.

The ratio of CD 4 to chemokine-receptor expression may also bean important factor regulating $T$-cell death, especially in infected cells or those contacted by X 4 HIV or X4env, respectively. Recently, several groups have described cell death by this CXCR4 mechanism in neurons and $T$ cells $(26,43,44)$. Therefore, this X4env/CXCR4 mechanism of death has profound implications for not only peripheral T-cell depletion, but also neuronal loss and AIDS-related dementia during the late stages of HIV infection. Another novel aspect of our results is theapparent protectiverolethat $C D 4$ may play in CXCR4-mediated death. Thewell-documented downregulation of CD 4 in HIV-infected cells may explain why 
membranebound X4env can interact easily with CXCR4 on the surface of theinfected T cell. In uninfected T cells, theupregulation of CXCR4, as shown recently to bethe case in T cells from lymphoid tissue in HIV-infected patients, may suffice to alter the CD 4/CXCR4 ratio to favor X4env/CXCR4 interactions.

The results of this study improves our understanding of the pathogenesis of HIV-mediated T-cell death. Specifically, wehaveshown that different death-prone signaling pathways are triggered by HIVenv interacting with its receptors and thereby clarifying the significant confusion existing as to how $T$ cells diein the context of HIV infection.

\section{Acknowledgments}

This work was supported by N IH grant R01 Al40384. The authors would liketo thank N.E. Vlahakis for technical support with confocal microscopy, C. Cicala and A. Fauci for providing us with 92Ug20.9 envelope protein, D. Littman for providing the HXB2 and JRFL gp160 plasmids, members of thePaya Laboratory for helpful discussions, and Teresa $\mathrm{H}$ off for manuscript preparation.

1. Cohen, J. 1993. What causes theimmunesystem collapseseen in AIDS? Science. 260:1256.

2.Zarling, J.M., et al. 1990. HIV-infected humans, but not chimpanzees, have circulating cytotoxic T lymphocytes that lyse uninfected CD4+cells. J. Immunol. 144:2992-2998.

3. N ardelli, B., Gonzalez, C. ., Schechter, M ., and Valentine, F.T. 1995. CD4+ blood lymphocytes are rapidly killed in vitro by contact with autologous human immunodeficiency virus-infected cells. Proc. Natl. Acad. Sci.USA 92:7312-7316.

4. Laurent-Crawford, A.G., et al. 1993. M embraneexpression of HIV envelope glycoproteins triggers apoptosis in CD 4 cells. AIDS Res. Hum. Retroviruses. 9:761-773.

5. Finkel, T.H., et al. 1995. Apoptosis occurs predominantly in bystander cells and not in productively infected cells of HIV- and SIV-infected lymph nodes. Nat. M ed. 1:129-134

6. Algeciras, A., D ockrell, D.H., Lynch, D.H., and Paya, C.V. 1998. CD 4 regulates susceptibility to Fas ligand- and tumor necrosisfactor-mediated apoptosis. J. Exp. Med. 187:711-720.

7. Westendorp, M .O., et al. 1995. Sensitization of T cells to CD95-mediated apoptosis by HIV-1 Tat and gp120. Nature. 375:497-500.

8. Corbeil, J., and Richman, D.D. 1995. Productiveinfection and subsequent interaction of CD 4-gp120 at the cellular membrane is required for HIVinduced apoptosis of CD4+T cells. J. Gen. Virol. 76:681-690.

9. Gandhi, R.T., et al. 1998. HIV-1 directly kills CD 4+T cells by a Fas-independent mechanism. J. Exp. M ed. 187:1113-1122.

10. Kolesnitchenko, V., et al. 1997. A major human immunodeficiency virus type 1-initiated killing pathway distinct from apoptosis. J. Virol. 71:9753-9763.

11. Penn, M .L., Grivel, J.C., Schramm, B., Goldsmith, M .A., and M argolis, L. 1999. CXCR4 utilization is sufficient to trigger CD 4+T cell depletion in HIV-1-infected human lymphoid tissue. Proc. Natl. Acad. Sci. USA. 96:663-668.

12. Asjo, B., et al. 1986. Replicativecapacity of human immunodeficiency virus from patients with varying severity of HIV infection. Lancet. 2:660-662.

13. Tersmette, M., et al. 1989. Evidence for a role of virulent human immunodeficiency virus (HIV) variants in the pathogenesis of acquired immunodeficiency syndrome: studies on sequential HIV isolates. J. Virol. 63:2118-2125.

14. Cheng-M ayer, C., Seto, D., Tateno, M., and Levy, J.A. 1988. Biologic features of HIV-1 that correlate with virulence in the host. Science. 240:80-82.

15. Roederer, M., Dubs, J.G., Anderson, M.T., Raju, P.A., and Herzenberg, L.A. 1995. CD 8 naiveT cell counts decrease progressively in HIV-infected adults. J. Clin. Invest. 95:2061-2066.

16. Herbein, G., et al. 1998. Apoptosis of CD8+ T cells is mediated by macrophages through interaction of HIV gp120 with chemokine receptor CXCR4. Nature. 395:189-194.

17. Harouse, J.M ., Gettie, A., Tan, R.C., Blanchard, J., and Cheng-M ayer, C. 1999. Distinct pathogenic sequela in rhesus macaques infected with CCR5 or CXCR4 utilizing SHIVs. Science. 284:816-819.

18. Algeciras-Schimnich, A., Griffith, T.S., Lynch, D.H., and Paya, C.V. 1999. Cell cycle-dependent regulation of FLIP levels and susceptibility to Fasmediated apoptosis. J. I mmunol. 162:5205-5211.

19. Wu, L., et al. 1996. CD 4-induced interaction of primary HIV-1 gp120 glycoproteins with the chemokine receptor CCR-5. Nature. 384:179-183.

20. Bandres, J.C., et al. 1998. Human immunodeficiency virus (HIV) envelope binds to CXCR4 independently of CD 4, and binding can be enhanced by interaction with solubleCD 4 or by HIV envelope deglycosylation. J. Virol. 72:2500-2504.

21. Hill, C.M., et al. 1997. Envelope glycoproteins from human immunodeficiency virus types 1 and 2 and simian immunodeficiency virus can use human CCR5 as a coreceptor for viral entry and makedirect CD 4-dependent interactions with this chemokine receptor. J. Virol. 71:6296-6304.

22. Sullivan, $N$., et al. 1998. CD4-induced conformational changes in the human immunodeficiency virus type $1 \mathrm{gp} 120$ glycoprotein: consequences for virus entry and neutralization. J. Virol. 72:4694-4703.

23. Bleul, C.C., et al. 1996. Thelymphocytechemoattractant SDF-1 is a ligand for LESTR/fusin and blocks HIV-1 entry. Nature. 382:829-833.

24. Oberlin, E., et al. 1996. The CXC chemokine SDF-1 is the ligand for LESTR/fusin and prevents infection by T-cell-line-adapted HIV-1 [erratum 1996, 384:288]. Nature. 382:833-835.

25. Amara, A., et al. 1997. HIV coreceptor downregulation as antiviral principle: SDF-1alpha-dependent internalization of the chemokine receptor CXCR4 contributes to inhibition of HIV replication. J. Exp. Med. 186:139-146.

26. Berndt, C., M opps, B., Angermuller, S., Gierschik, P., and Krammer, P.H. 1998. CXCR4 and CD 4 mediate a rapid CD 95-independent cell death in CD 4(+) T cells. Proc Natl. Acad. Sci. USA 95:12556-12561.

27. Su, S.B., et al. 1999. Inhibition of tyrosine kinase activation blocks the down-regulation of CXC chemokinereceptor 4 by HIV-1 gp120 in CD 4+T cells. J. Immunol. 162:7128-7132.

28. Madani, N ., Kozak, S.L., Kavanaugh, M .P., and Kabat, D . 1998. gp120 enve lope glycoproteins of human immunodeficiency viruses competitively antagonizesignaling by coreceptors CXCR4 and CCR5. Proc. Natl. A cad. Sci. USA. 95:8005-8010.

29. Andersson, J., et al. 1998. Early reduction of immune activation in lymphoid tissuefollowing highly activeHIV therapy. AIDS. 12:F123-F129.

30. Suzuki, Y., et al. 1999. Determinant in human immunodeficiency virustype 1 for efficient replication under cytokineinduced CD 4(+) T-helper 1 (Th1)and Th2-typeconditions. J. Virol. 73:316-324.

31. Jourdan, P., et al. 1998. IL-4 induces functional cell-surface expression of CXCR4 on human T cells [erratum 1999, 162:3105]. J. Immunol. 160:4153-4157.

32. Orsini, M.J., Parent, J.L., M undell, S.J., and Benovic, J.L. 1999. Trafficking of the HIV coreceptor CXCR4. Role of arrestins and identification of residues in thec-terminal tail that mediatereceptor internalization. J. Biol. Chem. 274:31076-31086.

33. Haribabu, B., et al. 1997. Regulation of human chemokine receptors CXCR4. Roleof phosphorylation in desensitization and internalization.J. Biol. Chem. 272:28726-28731.

34. Hoxie, J.A., et al. 1986. Alterations in T4 (CD4) protein and mRNA synthe sis in cells infected with HIV. Science. 234:1123-1127.

35. Littman, D.R. 1996. TheCD 4 molecule Roles in T Iymphocytes and in HIV disease Introduction. Curr. Top. Microbiol. Immunol. 205: $\mathrm{v}-\mathrm{X}$.

36. Garcia, J.V., and M iller, A.D. 1991. Serine phosphorylation-independent downregulation of cell-surface CD 4 by nef. Nature. 350:508-511.

37. Cicala, C., et al. 2000. HIV-1 envelope induces activation of caspase-3 and cleavage of focal adhesion kinase in primary human CD4(+) T cells. Proc. Natl. Acad. Sci. USA. 97:1178-1183.

38. Pantaleo, G., et al. 1993. HIV infection is activeand progressivein lymphoid tissue during the clinically latent stage of disease. Nature. 362:355-358.

39. Fox, C.H., et al. 1991. Lymphoid germinal centers are reservoirs of human immunodeficiency virus type1 RN A. J. Infect. Dis. 164:1051-1057.

40. Sunila, I., Vaccarezza, M ., Pantaleo, G., Fauci, A.S., and Orenstein, J.M . 1997 gp120 is present on the plasma membrane of apoptotic CD 4 cells prepared from lymph nodes of HIV-1-infected individuals: an immunoelectron microscopic study. AIDS. 11:27-32.

41. Rosok, B.I., et al. 1998. Correlates of apoptosis of CD 4+and CD8+T cells in tonsillar tissue in HIV type 1 infection. AIDS Res. H um. Retroviruses. 14:1635-1643.

42. Cotton, M.F., et al. 1997. Apoptosis of CD4+and CD8+T cells isolated immediately ex vivo correlates with disease severity in human immunodeficiency virus type 1 infection. Pediatr. Res. 42:656-664.

43. Hesselgesser, J., et al. 1998. N euronal apoptosis induced by HIV-1 gp120 and the chemokine SDF-1 al pha is mediated by the chemokine receptor CXCR4. Curr. Biol. 8:595-598.

44. Baird-Piechaczyk, M., Robert-H ebmann, V., Roland, J., Coudronniere, N., and Devaux, C. 1999. Role of CXCR4 in HIV-1-induced apoptosis of cells with a CD4+, CXCR4+ phenotype. Immunol. Lett. 70:1-3. 